\title{
Estimating staffing requirements using workload indicators of staffing need at Braun District Hospital in Morobe Province, Papua New Guinea
}

\author{
Dixon Dimiri ${ }^{*}$ (D), Nelson Mek ${ }^{1}$, Mary Therese Apini ${ }^{1}$, Thelma Ali ${ }^{1}$, Grace Turi Pumuye ${ }^{1}$, Varage John Laka ${ }^{1}$, \\ Rosemary Jogo ${ }^{1}$, Pamela Kari ${ }^{1}$, Deki ${ }^{2}$, Okech Mollent ${ }^{2}$, Dapeng Luo ${ }^{2}$, Anna Maalsen², Katu Yapi ${ }^{3}$ and \\ Robin Madodo ${ }^{3}$
}

\begin{abstract}
Background: Papua New Guinea has seen some improvements in health indicators over the past years, but the pace of improvements is not as robust as expected. The Health Services Plan for Braun District Hospital redevelopment identified the importance of reflecting the hospital's role in the broader health system, particularly in upgrading the services to service a bigger population. In August 2020, the hospital was upgraded from a health centre-level 3 to a district hospital level 4 . The need for assessing human resources for health requirements for this level of care was thus necessary.

Methods: The National Department of Health approved the use of the workload indicators of staffing need as the best tool to support in estimating staff requirements for the newly upgraded hospital. The focus was on clinical and non-clinical staff. Using already developed workload components and activity standards by the expert working groups for level 4 facilities, we visited the facility and collected data through interviews with the Lutheran Health Services representative, hospital management and staff. The technical task force reviewed daily registers, monthly reports and the data in the electronic national health information systems. The information collected was analysed using the workload indicators of staffing need software and interpreted.

Results: There were staffing shortages among the clinical staff like the medical officers, nursing officers, health extension officers, pharmacists, radiology staff unit and in the laboratory staff. Shortages among the non-clinical staff were recorded by the cashiers, security officers, drivers and boat skippers. The results showed that the facility lacks a medical laboratory technologist, pharmacists and a medical imaging technologist. The community health workers in this facility are utilized in all the areas where shortages are registered to multitask.

Conclusion: The results from this WISN study provide evidence for basing staffing decisions on. The WISN results from Braun District Hospital show that the facility requires a total of 33 inpatient nurses against the existing 21 inpatient nurses thus giving a staff gap of -12 and a WISN ratio of 0.67 . It is thus recommended that the hospital management prioritizes recruitment of nurses or if no resources, reassign one of the outpatient nurses to alleviate the
\end{abstract}

*Correspondence: dixon_dimiri@health.gov.pg; dixondimiri@gmail.com

${ }^{1}$ Health Workforce Standards and Accreditation Branch, National

Department of Health, Port Moresby, Papua New Guinea

Full list of author information is available at the end of the article

(c) The Author(s) 2021. Open Access This article is licensed under a Creative Commons Attribution 4.0 International License, which permits use, sharing, adaptation, distribution and reproduction in any medium or format, as long as you give appropriate credit to the original author(s) and the source, provide a link to the Creative Commons licence, and indicate if changes were made. The images or other third party material in this article are included in the article's Creative Commons licence, unless indicated otherwise in a credit line to the material. If material is not included in the article's Creative Commons licence and your intended use is not permitted by statutory regulation or exceeds the permitted use, you will need to obtain permission directly from the copyright holder. To view a copy of this licence, visit http://creativecommons.org/licenses/by/4.0/. The Creative Commons Public Domain Dedication waiver (http://creativeco mmons.org/publicdomain/zero/1.0/) applies to the data made available in this article, unless otherwise stated in a credit line to the data. 
pressure among the inpatient nurses or the extra theatre nurses to offer some services in the inpatient wards. WISN results can help managers make decisions such as change of health facility status from a health centre to a district hospital.

Keywords: Clinical staff, Non-clinical staff, Workload indicators of staffing need

\section{Background}

Papua New Guinea (PNG) has a government-funded health system throughout the country. It is supplemented by government-subsidized health services provided by various Christian missions such the Lutheran Health services. The churches are a major partner providing 50\% of the health care services mostly in the rural areas [1]. The 2007 Provincial Health Authority Act [2, 3] enabled the provincial government to establish Provincial Health Authorities (PHAs) responsible for both primary and secondary health care in the provinces. This legislation streamlines the provision of health services at the provincial level and brings together the provincial health departments, hospitals, and district health services under one management board.

The Health Plan [4] was developed to strengthen primary health care (for all) and improve service delivery to the rural majority and the urban disadvantaged and ensure quality health care services are readily accessible and affordable for all. The health services are organized into six levels of care namely the village health posts, community health posts, health centres, district hospitals, provincial hospitals and finally the national referral hospital [5-7].

The NDoH, Medical Standards Division, Workforce Standards and Accreditation Branch (WSA) have the mandate of streamlining workforce standards for the country to enable achievement of the Universal Health Coverage (UHC) and improve health outcomes in the country towards achievement of national and global health commitments [8]. Despite achieving considerable success through the multi-sectoral approach in strengthening the human resources for health capacities, there still exist some gaps such as imbalances in the development and distribution of health workers. In addition to shortages of qualified health professionals in underserved areas, there are significant variations in health services and their quality which causes inequities in health outcomes [9].

With the support of WHO, the WSA branch is working closely with PHAs and health facilities at various levels to advance long term solutions to improve health workers numbers distribution and utilization to ensure quality care for all. The country has integrated the use of workload indicators of staffing need as the evidence-based method for adoption for health worker planning [10].
From the early 1960s, Braun was a health centre serving a small island community which has since grown and services have equally expanded. It is run by Lutheran Health Services (LHS) and located in Finschafen District, Morobe Province in Momase Region of PNG. The hospital status was upgraded from Level 3 to Level 4 in August 2020. The facility has a bed capacity of 120 beds and serves a greater population with a staff capacity of 56 both clinical and non-clinical staff. The upgrading of Braun District Hospital was deemed necessary to ease referral for the primary care facilities in Morobe Province and decongest the provincial and regional facilities.

The overall goal of this study was to determine staffing requirements based on workloads for improved health services in the new upgraded hospital. The results would guide the hospital management board and sponsors on optimum numbers of health workers required per cadre for improved quality health services using WISN.

\section{Methods}

The WHO WISN methodology was introduced and approved for use in the country in 2017. The WSA team was trained as the Technical Task Force (TTF) to lead the process of WISN in the country. Expert working groups (EWGs) for all the cadres were trained and later develop the workload components for all the cadres and the corresponding activity standards for adoption in the country. The workload components were validated, approved and printed for use in the country. Available working time (AWT) for the facility for each of the cadres was established. Using cadre-specific workload components, the TTF visited Braun District Hospital to gather health service statistics for 2019 for use in the WISN software. The results generated from the software were analysed and interpreted. Staff interviews, management and LHS administration were also conducted to enable further understanding. Other sources of health service statistics were the daily registers and the partial health information systems.

\section{Setting of the study}

The study was set in Braun District hospital in Finschafen District, Morobe Province in the wider region of Momase in Papua New Guinea (PNG). The study focused on all the staff of Braun District Hospital. It included both the 


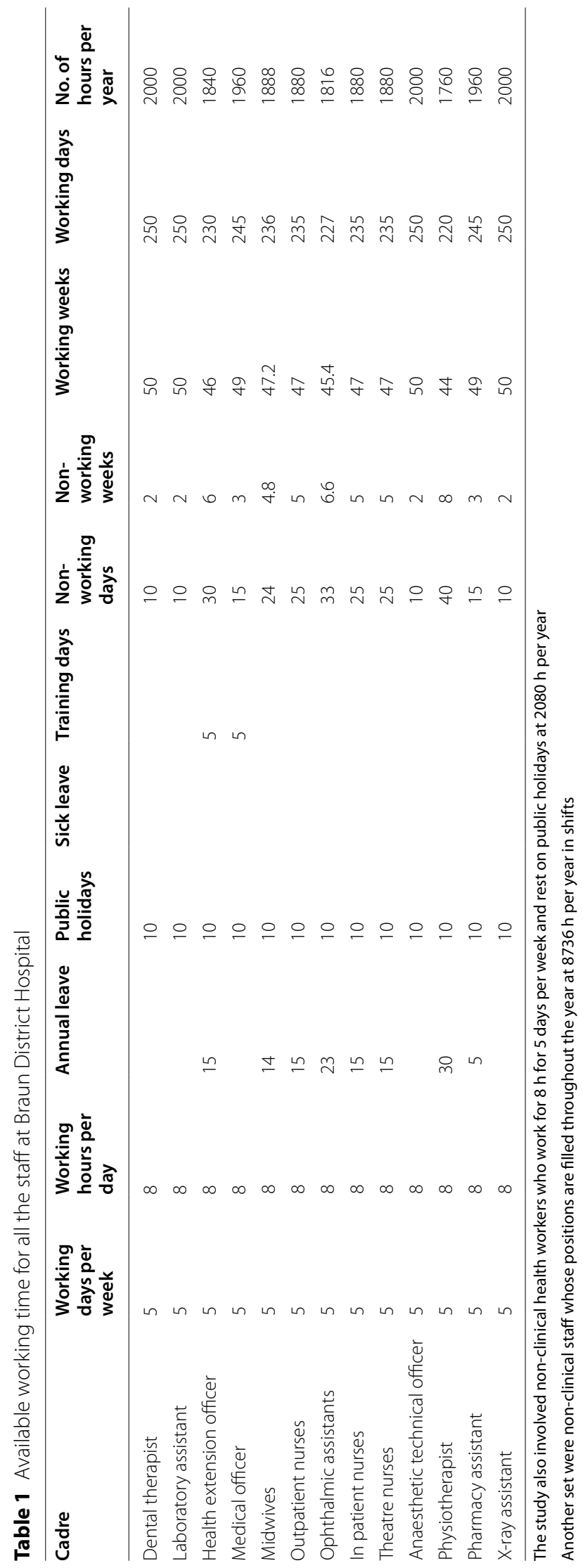


clinical and non-clinical staff in all the service areas of the hospital.

\section{Sampling design, size and procedure}

This was a case-specific analysis for Braun District Hospital. Both clinical and non-clinical staff were included in the study. The clinical staff included: medical officers, nursing officers, midwives, health extension officers, community health workers, ophthalmic assistant, dental therapist, medical laboratory assistants, X-ray assistant, physiotherapist and pharmacy assistants. The non-clinical staff included: the hospitals' health administrator, statistician, accountant, accounts clerk, cashier, key board operator, electrician, plumber, drivers, boat skippers, security officers and the morgue attendant.

The TTF held an initial meeting with all the service areas managers in the hospital to orient them on the agenda of the week to avoid disruption of the hospital services. The daily registers submitted provided the activities conducted and the records of each of the services. The human resource manager from LHS provided all the administrative records that supported the WISN study such as staff numbers, information on staff absences mainly the annual, training and sick leaves. An overview of the HRH management practices implemented by the hospitals under LHS was highlighted. This information was critical in the establishment of AWT for each of the cadres and shift programmes in the facility. Further, information about the services offered in the facility, service areas and current staffing based on the workload components developed for each cadre was collected. The hospital statistician shared the monthly and annual statistics from the electronic National Health Information System (eNHIS) which had data for some key indicators and not all services as listed in the data collection sheet. Data verification and validation were conducted before being uploaded into the WISN software.

\section{Results}

For each of the cadres, AWT was established as shown in Table 1. The AWT ranged from 1760 to $2000 \mathrm{~h}$ for year of study. Both the inpatient and outpatient nurses registered an AWT of $1880 \mathrm{~h}$ each while the medical officer had a total of $1960 \mathrm{~h}$. The health extension officer registered a total of $1840 \mathrm{~h}$ as AWT. While the dental therapist, medical laboratory assistants and the X-ray assistants all recorded $2000 \mathrm{~h}$ as AWT. The physiotherapist had an AWT of $1760 \mathrm{~h}$ for the year and the midwives had an AWT of $1888 \mathrm{~h}$ for the year 2019. The non-clinical staff worked under 2 types of shifts arrangements. There were those who worked for $8 \mathrm{~h}$ per day for 5 days in a week and rested on all the public holidays like the accountant, health administrator, statistician, accounts clerk, cashier and the key board operator. Drivers, boat skippers, security officers worked for $24 \mathrm{~h}$ per day, 7 days a week throughout the year. Their posts had to be filled throughout.

Still on Table 1, the results show that the facility had no records on the sick leaves from all the cadres. On training, only the medical officer and the health extension officer had at least 5 days each for training while other cadres had no training throughout the year. As for the annual leave which is a statutory requirement for all the cadres, not all were able to take the annual leaves. They included the dental therapist, laboratory assistant, medical officer, anaesthetic technical officer and the X-ray assistant.

The workload components for each of the cadres and their corresponding activity/service standards for the three workload groups namely: health, support and additional activities were provided for each of the cadres. Table 2 provides the three workload groups, service standards and annual statistics collected in the facility for the 13 clinical cadres under study.

The workload components, activity standards and the annual statistics were first uploaded in the WISN software to provide staffing requirements for health services also known as workload group 1. This was followed by uploading the support activities or workload group 2 activities and the category allowances to get the category allowance factor in percentage and a staff requirement for the support activities. Finally, additional activities or workload group 3 activities were uploaded into the software with the relevant additional allowances to provide the individual allowance standards in hours per year and the individual allowance factor which is the staff required undertaking additional activities. Using the WISN formula for calculating staff requirements of $1 \times 2+3=$ staff requirements, the staffing requirement per cadre were calculated as summarized in Table 3.

Table 3 shows mixed results of balances, shortages and cases of staff surpluses. Balances are recorded among the midwives, physiotherapist and the dental therapist. The existing staff equal the WISN-calculated staffing requirements hence recording a difference of 0 and a WISN ratio of 1.00 .

Staffing shortages were recorded across 6 cadres. The staff differences with a negative sign mean a gap or a shortage. The medical laboratory assistants have 2 existing staff and the staffing requirement calculated by WISN is 3 giving a difference of -1 and WISN ratio of 0.67 . The medical officers have an existing staff of 1 and the calculated requirement based on workloads is 4 thus a difference of at -3 and WISN ratio of 0.25 . The ophthalmic assistants have an existing staff of 1 and the WISN requirement estimates a total of 3 staff thus a gap 
Table 2 Workload components and activity standards and annual statistics collected

\begin{tabular}{|c|c|c|}
\hline \multicolumn{3}{|l|}{ Medical officers } \\
\hline Workload components & Service standards & Annual-2019 \\
\hline \multicolumn{3}{|c|}{ Health service activities (Group 1 activities) } \\
\hline Consultations & 20 min per patient & 5880 \\
\hline Admissions & 30 min per patient & 3920 \\
\hline Ward rounds & 20 min per patient & 3920 \\
\hline Minor surgeries & 17 min per patient & 6918 \\
\hline Intermediate surgeries & 45 min per patient & 2613 \\
\hline Major surgeries & 259 min per patient & 454 \\
\hline Medical procedures & 30 min per patient & 3920 \\
\hline Complex deliveries & 60 min per patient & 520 \\
\hline Referrals & 15 min per patient & 321 \\
\hline Assault and injuries & 30 min per patient & 1840 \\
\hline Death declaration & 10 min per patient & 35 \\
\hline Discharge & 10 min per patient & 5524 \\
\hline Re-attendances & 15 min per patient & 11,760 \\
\hline \multicolumn{3}{|l|}{ Support activities (Group 2 activities) } \\
\hline Morning devotion & 45 min per day & \\
\hline Daily debriefs & 20 min per day & \\
\hline Clinical case presentations & $1 \mathrm{~h}$ per week & \\
\hline Meeting of paramedics & $1 \mathrm{~h}$ per month & \\
\hline Staff meetings & $12 \mathrm{~h}$ per year & \\
\hline Continuous medical education & $24 \mathrm{~h}$ per year & \\
\hline Outreaches & 20 days/year & \\
\hline \multicolumn{3}{|c|}{ Additional activities (Group 3 activities) } \\
\hline Staff supervision & $12 \mathrm{~h}$ per year & \\
\hline Supervision of students & 16 h per year & \\
\hline General administration & 30 min per day & \\
\hline Surveillance reporting & $32 \mathrm{~h}$ per year & \\
\hline Monthly reports & $12 \mathrm{~h}$ per year & \\
\hline Medical legal duties & $8 \mathrm{~h} /$ year & \\
\hline
\end{tabular}

\section{Dental therapists}

\begin{tabular}{|c|c|c|c|}
\hline Health service/group 1 & No. per year & Service standard/unit & $\begin{array}{l}\text { Standard } \\
\text { workload }\end{array}$ \\
\hline Dental examinations & 340 & $15 \mathrm{~min} /$ patient & 8000 \\
\hline Referrals & 20 & $10 \mathrm{~min} /$ patient & 12,000 \\
\hline Tooth extractions & 340 & $45 \mathrm{~min} /$ patient & 2667 \\
\hline Temporary dressings & 18 & $30 \mathrm{~min} /$ patient & 4000 \\
\hline Patient management & 340 & $45 \mathrm{~min} /$ patient & 2667 \\
\hline \multicolumn{4}{|l|}{ 2. Laboratory assistant } \\
\hline Malaria smear & 3261 & $25 \mathrm{~min} / \mathrm{sample}$ & 4800 \\
\hline Malaria RDT & 515 & $10 \mathrm{~min} / \mathrm{sample}$ & 12,000 \\
\hline White cell count & 3154 & $12 \mathrm{~min} / \mathrm{sample}$ & 10,000 \\
\hline WBC differential & 194 & $28 \mathrm{~min} / \mathrm{sample}$ & 4286 \\
\hline ESR & 146 & $15 \mathrm{~min} / \mathrm{sample}$ & 8000 \\
\hline Platelet count & 17 & $12 \mathrm{~min} / \mathrm{sample}$ & 10,000 \\
\hline Total lymphocyte count & 19 & $12 \mathrm{~min} / \mathrm{sample}$ & 10,000 \\
\hline VDRL & 353 & $10 \mathrm{~min} / \mathrm{sample}$ & 12,000 \\
\hline TPHA & 438 & $10 \mathrm{~min} / \mathrm{sample}$ & 12,000 \\
\hline
\end{tabular}


Table 2 (continued)

\begin{tabular}{|c|c|c|c|}
\hline \multicolumn{4}{|l|}{ Dental therapists } \\
\hline Health service/group 1 & No. per year & Service standard/unit & $\begin{array}{l}\text { Standard } \\
\text { workload }\end{array}$ \\
\hline HIV testing & 182 & $20 \mathrm{~min} / \mathrm{sample}$ & 6000 \\
\hline Hepatitis B & 242 & $20 \mathrm{~min} / \mathrm{sample}$ & 6000 \\
\hline Widal test & 46 & $20 \mathrm{~min} / \mathrm{sample}$ & 6000 \\
\hline Pregnancy test & 69 & $5 \mathrm{~min} / \mathrm{sample}$ & 24,000 \\
\hline Urinalysis strip & 396 & $5 \mathrm{~min} / \mathrm{sample}$ & 24,000 \\
\hline Urinalysis microscopy & 136 & $10 \mathrm{~min} / \mathrm{sample}$ & 12,000 \\
\hline Blood fluid split & 17 & $5 \mathrm{~min} / \mathrm{sample}$ & 24,000 \\
\hline Blood fluid microscopy & 15 & $10 \mathrm{~min} / \mathrm{sample}$ & 12,000 \\
\hline AFB & 854 & $35 \mathrm{~min} / \mathrm{sample}$ & 3429 \\
\hline Other body fluids & 36 & $10 \mathrm{~min} / \mathrm{sample}$ & 12,000 \\
\hline Gram stain & 32 & $10 \mathrm{~min} / \mathrm{sample}$ & 12,000 \\
\hline Blood grouping & 719 & $10 \mathrm{~min} / \mathrm{sample}$ & 12,000 \\
\hline Cross match & 250 & $15 \mathrm{~min} / \mathrm{sample}$ & 8000 \\
\hline Blood collection & 173 & $20 \mathrm{~min} / \mathrm{sample}$ & 6000 \\
\hline $\mathrm{BSL}$ & 79 & $7 \mathrm{~min} / \mathrm{sample}$ & 17,143 \\
\hline Stool for OCP & 10 & $8 \mathrm{~min} / \mathrm{sample}$ & 15,000 \\
\hline CSF exam & 1 & $9 \mathrm{~min} / \mathrm{sample}$ & 13,333 \\
\hline Alkaline phosphate & 57 & $6 \mathrm{~min} / \mathrm{sample}$ & 20,000 \\
\hline Amylase & 14 & $6 \mathrm{~min} / \mathrm{sample}$ & 20,000 \\
\hline Cholinesterase & 6 & $6 \mathrm{~min} / \mathrm{sample}$ & 20,000 \\
\hline Gamma glutamyl & 58 & $6 \mathrm{~min} / \mathrm{sample}$ & 20,000 \\
\hline Aspartate A & 75 & $6 \mathrm{~min} / \mathrm{sample}$ & 20,000 \\
\hline Alanine Amino & 63 & $6 \mathrm{~min} / \mathrm{sample}$ & 20,000 \\
\hline Leucine Amino & 5 & $6 \mathrm{~min} / \mathrm{sample}$ & 20,000 \\
\hline Albumin & 61 & $6 \mathrm{~min} / \mathrm{sample}$ & 20,000 \\
\hline Blood urea nitrogen & 81 & $6 \mathrm{~min} / \mathrm{sample}$ & 20,000 \\
\hline Calcium & 7 & $6 \mathrm{~min} / \mathrm{sample}$ & 20,000 \\
\hline Creatine & 116 & $6 \mathrm{~min} / \mathrm{sample}$ & 20,000 \\
\hline Direct bilirubin & 10 & $6 \mathrm{~min} / \mathrm{sample}$ & 20,000 \\
\hline Total bilirubin & 49 & $6 \mathrm{~min} / \mathrm{sample}$ & 20,000 \\
\hline Total cholesterol & 1 & $6 \mathrm{~min} / \mathrm{sample}$ & 20,000 \\
\hline Triglyceride & 9 & $6 \mathrm{~min} / \mathrm{sample}$ & 20,000 \\
\hline Total protein & 37 & $6 \mathrm{~min} / \mathrm{sample}$ & 20,000 \\
\hline Uric acid & 81 & $6 \mathrm{~min} / \mathrm{sample}$ & 20,000 \\
\hline Sodium & 99 & $6 \mathrm{~min} / \mathrm{sample}$ & 20,000 \\
\hline Potassium & 159 & $6 \mathrm{~min} / \mathrm{sample}$ & 20,000 \\
\hline Chloride & 74 & $6 \mathrm{~min} / \mathrm{sample}$ & 20,000 \\
\hline C-reactive protein & 4 & $6 \mathrm{~min} / \mathrm{sample}$ & 20,000 \\
\hline Hepatitis C & 25 & $20 \mathrm{~min} / \mathrm{sample}$ & 6000 \\
\hline \multicolumn{4}{|l|}{ 3.Health Extension Officer } \\
\hline TB consultations & 160 & $30 \mathrm{~min} /$ patient & 3680 \\
\hline Deaths & 2 & $10 \mathrm{~min} /$ patient & 11,040 \\
\hline Patient reviews & 160 & $120 \mathrm{~min} /$ patient & 920 \\
\hline Admission & 160 & $20 \mathrm{~min} /$ patient & 5520 \\
\hline In patient care for TB & 160 & $20 \mathrm{~min} /$ patient & 5520 \\
\hline Ward rounds & 1443 & $20 \mathrm{~min} /$ patient & 5520 \\
\hline Dispensing of TB drugs & 160 & $7 \mathrm{~min} /$ patient & 15,771 \\
\hline
\end{tabular}


Table 2 (continued)

\begin{tabular}{|c|c|c|c|}
\hline \multicolumn{4}{|l|}{ Dental therapists } \\
\hline Health service/group 1 & No. per year & Service standard/unit & $\begin{array}{l}\text { Standard } \\
\text { workload }\end{array}$ \\
\hline Referrals & 2 & $10 \mathrm{~min} /$ patient & 11,040 \\
\hline Discharges & 156 & $20 \mathrm{~min} /$ patient & 5520 \\
\hline Assist in minor surgeries & 401 & $17 \mathrm{~min} /$ patient & 6494 \\
\hline \multicolumn{4}{|l|}{ 4. Medical Officer } \\
\hline Consultations & 1,784 & $20 \mathrm{~min} /$ patient & 5880 \\
\hline Admissions & 1143 & $30 \mathrm{~min} /$ patient & 3920 \\
\hline Ward rounds & 1143 & $20 \mathrm{~min} /$ patient & 5880 \\
\hline Minor surgical procedures & 401 & $17 \mathrm{~min} /$ patient & 6918 \\
\hline Intermediate surgeries & 319 & $45 \mathrm{~min} /$ patient & 2613 \\
\hline Major surgeries & 70 & $259 \mathrm{~min} /$ patient & 454 \\
\hline Medical procedures & 82 & $30 \mathrm{~min} /$ patient & 3920 \\
\hline Complex deliveries & 31 & $60 \mathrm{~min} /$ patient & 1960 \\
\hline Referrals & 251 & $15 \mathrm{~min} /$ patient & 7840 \\
\hline Assault and injuries & 136 & $30 \mathrm{~min} /$ patient & 3920 \\
\hline Death declaration & 38 & $10 \mathrm{~min} /$ patient & 11,760 \\
\hline Discharge & 1401 & $10 \mathrm{~min} /$ patient & 11,760 \\
\hline Re-attendances & 7853 & $15 \mathrm{~min} /$ patient & 7840 \\
\hline \multicolumn{4}{|l|}{ 5. Midwives } \\
\hline Admissions & 335 & $20 \mathrm{~min} /$ patient & 5664 \\
\hline Nursing care & 335 & $600 \mathrm{~min} /$ patient & 189 \\
\hline Labour management & 335 & $240 \mathrm{~min} /$ patient & 472 \\
\hline Ward rounds & 335 & $20 \mathrm{~min} /$ patient & 5664 \\
\hline Normal deliveries & 335 & $45 \mathrm{~min} /$ patient & 2517 \\
\hline Immediate Newborn care & 362 & $35 \mathrm{~min} /$ patient & 3237 \\
\hline Newborn care & 364 & $15 \mathrm{~min} /$ patient & 7552 \\
\hline Post- natal care & 362 & $20 \mathrm{~min} /$ patient & 5664 \\
\hline Discharges & 364 & $10 \mathrm{~min} /$ patient & 11,328 \\
\hline Care of the dead & 2 & $30 \mathrm{~min} /$ patient & 3776 \\
\hline \multicolumn{4}{|l|}{ 6. Nurses_outpatient } \\
\hline Antenatal clinic & 352 & $40 \mathrm{~min} /$ patient & 2820 \\
\hline Subsequent visit & 420 & $25 \mathrm{~min} /$ patient & 4512 \\
\hline Immunization injections & 6176 & $25 \mathrm{~min} /$ patient & 4512 \\
\hline Immunization oral & 2348 & $15 \mathrm{~min} /$ patient & 7520 \\
\hline Family planning-condoms & 8 & $10 \mathrm{~min} /$ patient & 11,280 \\
\hline Family planning-injection & 309 & $20 \mathrm{~min} /$ patient & 5640 \\
\hline Family planning-insertible & 156 & $30 \mathrm{~min} /$ patient & 3760 \\
\hline Family planning-natural & 15 & $25 \mathrm{~min} /$ patient & 4512 \\
\hline Family planning —oral & 21 & $10 \mathrm{~min} /$ patient & 11,280 \\
\hline Removal of implants & 2108 & $25 \mathrm{~min} /$ patient & 4512 \\
\hline Malnutrition management & 240 & $25 \mathrm{~min} /$ patient & 4512 \\
\hline Well baby clinic & 240 & $25 \mathrm{~min} /$ patient & 4512 \\
\hline \multicolumn{4}{|l|}{ 7. Ophthalmic assistant } \\
\hline OPD consultations & 5284 & $20 \mathrm{~min} /$ patient & 5448 \\
\hline Review of patients & 796 & $10 \mathrm{~min} /$ patient & 10,896 \\
\hline Assessment of refractions & 579 & $25 \mathrm{~min} /$ patient & 4358 \\
\hline Glass prescriptions & 579 & $5 \mathrm{~min} /$ patient & 21,792 \\
\hline Assistant in cataract surgery & 182 & $20 \mathrm{~min} /$ patient & 5448 \\
\hline
\end{tabular}


Table 2 (continued)

\section{Dental therapists}

Health service/group

No. per year

Service standard/unit

Standard

workload

Assessment of cataracts 182

Referrals 104

Removal of foreign objects $\quad 16$

Fundoscopy 16

Eye irrigation 16

Application of ointment $\quad 5284$

Post-cataract counselling $\quad 220$

8. Nurse inpatient

Management of TB 160

Care of the dead 39

Medical procedures 2866

Discharges 2866

Formula preparation $\quad 120$

Burns 5

Post-operative care $\quad 763$

Referrals 141

Wound dressing 20

Blood transfusion $\quad 35$

Pre-operative care $\quad 763$

Ward rounds 2866

OPD assessment $\quad 5928$

Admissions 2866

Administration of medications $\quad 5928$

Review of patients 4650

Sample collection 1076

Nursing care 2866

9. Theatre nurse

Minor surgical procedures $\quad 384$

Intermediate surgeries $\quad 309$

Major surgeries

10. Anaesthetic Technical Officer Minor surgical procedures

Intermediate surgeries

Major surgeries

11. Physiotherapist

Assessment of patients

Review of patients—wards

Ward rounds

Management of adults

Management of minors

Manual interventions

Mobility training

Deep breathing exercises

24

12. Pharmacy assistant

Dispensing of dangerous drugs $\quad 471$

Medicines dispensing

7313

7313

Pre-packaging services

$35 \mathrm{~min} /$ patient

$15 \mathrm{~min} /$ patient

$10 \mathrm{~min} /$ patient

$15 \mathrm{~min} /$ patient

$20 \mathrm{~min} /$ patient

$10 \mathrm{~min} /$ patient

$25 \mathrm{~min} /$ patient

$25 \mathrm{~min} /$ patient

$35 \mathrm{~min} /$ patient

$45 \mathrm{~min} /$ patient

$20 \mathrm{~min} /$ patient

$50 \mathrm{~min} /$ patient

$75 \mathrm{~min} /$ patient

$35 \mathrm{~min} /$ patient

$30 \mathrm{~min} /$ patient

$74 \mathrm{~min} /$ patient

$30 \mathrm{~min} /$ patient

$45 \mathrm{~min} /$ patient

$75 \mathrm{~min} /$ patient

$20 \mathrm{~min} /$ patient

$20 \mathrm{~min} /$ patient

$25 \mathrm{~min} /$ patient

$20 \mathrm{~min} /$ patient

$20 \mathrm{~min} /$ patient

$600 \mathrm{~min} /$ inpatient day

$17 \mathrm{~min} /$ patient

$45 \mathrm{~min} /$ patient

$259 \mathrm{~min} /$ patient

$17 \mathrm{~min} /$ patient

$45 \mathrm{~min} /$ patient

$259 \mathrm{~min} /$ patient

$60 \mathrm{~min} /$ patient

$45 \mathrm{~min} /$ patient

$10 \mathrm{~min} /$ patient

$50 \mathrm{~min} /$ patient

$35 \mathrm{~min} /$ patient

$60 \mathrm{~min} /$ patient

$45 \mathrm{~min} /$ patient

$30 \mathrm{~min} /$ patient

$25 \mathrm{~min} /$ patient

$17 \mathrm{~min} /$ patient
$74 \mathrm{~min} /$ patient
3113

7264

10,896

7264

5448

10,896

4358

4685

3346

2603

6856

2342

1562

3346

3904

1583

3904

2603

1562

5856

5856

4685

5856

5856

195

6635

2507

436

7059

2667

463

1760

2347

10,560

2112

3017

1427

1760

2347

3920

4704

6918 
Table 2 (continued)

\begin{tabular}{llll}
\hline Dental therapists & No. per year & Service standard/unit & $\begin{array}{l}\text { Standard } \\
\text { workload }\end{array}$ \\
\hline Health service/group 1 & 363 & $40 \mathrm{~min} /$ patient & \\
\hline $\begin{array}{l}\text { Compounding } \\
\text { 13. X-ray assistant }\end{array}$ & 3105 & $30 \mathrm{~min} /$ patient & 4040 \\
Plain radiographs & 62 & $45 \mathrm{~min} /$ patient & 2600 \\
ECG & & & 4 \\
\hline
\end{tabular}

Table 3 WISN-calculated staffing requirements clinical staff at Braun District Hospital

\begin{tabular}{lcccc}
\hline Type of staff & Existing staff & Calculated requirement & Difference & WISN ratio \\
\hline Dental therapists & 1 & 1 & 0 & 1.00 \\
Medical laboratory assistants & 2 & 3 & -1 & 0.67 \\
Health extension officers & 3 & 1 & -3 & 3.00 \\
Medical officers & 1 & 4 & 0 & 0.25 \\
Midwives & 6 & 6 & 1 & 1.00 \\
Outpatient nurses & 5 & 4 & -2 & 1.25 \\
Ophthalmic assistants & 1 & 3 & -12 & 0.33 \\
In patient nurses & 21 & 33 & 3 & 0.64 \\
Theatre nurses & 4 & 1 & 1 & 0 \\
Anaesthetic technical staff & 2 & 1 & -2 & 2.00 \\
Physiotherapists & 1 & 1 & -1 & 1.00 \\
Pharmacy assistants & 2 & 4 & 0.50 \\
X-ray assistants & 1 & 2 & 0.50 \\
\hline
\end{tabular}

of -2 and WISN ratio of 0.33 . Also, the existing inpatient nurses are 21 and the estimated WISN-calculated requirements is 33 showing a difference of -12 and WISN ratio of 0.64 . Braun Hospital currently has 2 pharmacy staff in the facility but based on the workload, the WISN estimates a requirement of 4 giving a difference of -2 and a corresponding WISN ratio of 0.50 . Finally, only $1 \mathrm{X}$-ray assistant exists in the facility and the WISN requirements estimates a total of 2 staff thus a difference of -1 with a WISN ratio of 0.50 .

There were circumstances where calculated WISN staff requirements were less than existing staff. Such were recorded by the health extension officers, theatre nurses and anaesthetic technical staff (ATO). The existing health extension officers in the facility at the time of study were 3. The WISN-calculated requirements were estimated at 1 with a WISN ratio of 3.00 hence an extra staff of +2 . On the other hand, the theatre nurse in Braun hospital were 4 and the calculated WISN requirement was estimated as 1 based on the workloads with a WISN ratio of 4.00 meaning a cadre surplus of +3 while the ATO existing staff were 2 and the calculated requirement was 1 showing a surplus of +1 with a WISN ratio of 2.00 .
There were other results also gathered that are beneficial to the upgraded hospital from the WISN study. During the field visit to the facility, it was observed that the unit had no physiotherapy equipment, the laboratory and the pharmacy would run out of stock of needed reagents, commodities and medication required in the facility. All these have an impact on the specific cadre workload. It was also noted that most of the staff assistants like the medical laboratory assistants, ophthalmic assistant, $\mathrm{X}$-ray assistant and pharmacy assistants were all community health workers (CHWs). This is an indication of staff sharing and shifting tasks where the $\mathrm{CHW}$ undertakes roles that are not their traditional roles.

Non-clinical staffing requirements were also estimated based on the shifting programmes. The facility had a category of non-clinical staff working for 5 days, $8 \mathrm{~h}$ per day with non-working days during public holidays. Such positions have $2080 \mathrm{~h}$ per year. The calculated staffing requirements for such posts estimated a requirement of 0.96 staff rounded up to 1 . On the other hand, there were positions that require staff throughout the year for $24 \mathrm{~h}$ daily, 7 days a week and 365 days. They operated in 2 shifts with a total of $8736 \mathrm{~h}$. Such positions require a total 
of 4.2 staff $(8736 / 2080)$ for shifting. In other words, 4 full-time staff working in shifts is recommended for such positions like of the security officers and the drivers. This covers the continuous shift work all through the year. It was noted some non-clinical health workers engaged in more than one role. For example, the statistician functions as the electrician and a driver at the same time.

\section{Discussion}

Upgrading a health facility requires evidence to provide the health managers with the right information to make decisions on staffing requirements and even necessary equipment to respond to the new status of the facility. Thus, the WISN results provide the staffing requirements for each of the cadres in the health facility. Some indicated shortages, other balances while others an indication of more staff than required. These results provide further insights to the health workforce decisions.

For those cadres that reported a difference of 0 and WISN ratio of 1 , the results were interpreted as having a staff balance meaning that the existing staff were just sufficient to offer the services of that facility within the professional standards of the country. Thus, no action was required but sustenance of the services. However, if the new status of the facility anticipates increased workloads, the results can still be used to estimate future staffing based on expected increase of services as reported by other studies $[11,12]$.

For those cadres that had results showing negative differences, it was interpreted as staff shortages that require prioritization or reassignment of available cadres in cases of scarce resources like it is the situation in PNG. If the facility has funds, staff hiring can be prioritized too. For example, in the case of inpatient nurses that registered a gap of -12 , the immediate decision administrative decision before staff hiring could be to reassign one of the surplus outpatient nurses to join the inpatient nurses and reduce the workload pressure of the inpatient nurses. Alternatively, assign some of the theatre nurses to support the inpatient nurses during low seasons or when no surgical procedure cases are booked. For the medical officer, a shortage of -3 staff was registered. The immediate action could be to assign the +2 surplus health extension officers to support in consulting at the outpatient department and only to allow the only medical officer to attend to more critical cases that require his expertise and reduce the high workload pressure.

The higher the shortages, the higher the workload pressures and shown by the ratios that are less than 1. It further means that the cadres could be working under pressure, thus services have possibilities of being compromised. On the contrary, the staff cadres whose WISN ratios were more than 1 and positive differences, it meant that the cadres in existence were more than what the facility required. There were also cases of surplus staff meaning the calculated estimated requirement was less than the existing staff. Positive differences indicate that the number of staff for specific cadre in the facility is more than the staff required to cope with the existing workload. It also means that the WISN ratios are more than 1, an indication of low workload pressures. The higher the surpluses in the differences, the higher the WISN ratio registered. Ultimately, the lower the workload pressure on the particular staff cadre. It could also imply that the quality of the services in that facility should be better than those with negative differences due to the existence of more staff. The existing staff should therefore offer the best services as they have no work pressure at all.

The results provide information that resonates with the National Health Services Standards that require health facilities to have the right staff, right equipment and technology. Level 4 health facilities or district hospitals are required to deliver medical, child health/paediatric, maternal and minor surgical services (including public health activities). They also provide clinical support services in pharmacy, pathology, anaesthetics and radiology [13]. Human resources for health play the most significant role in delivering all planned health services in any facility and thus become a key priority to policy makers [14]. It is therefore necessary that they are well sourced, equipped and managed for them to effectively function. They must be in their right numbers, skill mix, right attitudes, skills and competencies and working in the right environment [15-17] to achieve the right health targets for the projected indicators.

Our study findings strengthen similar studies that show inequities in staff distribution even within facilities [18]. We provide evidence on further uses of WISN results in making management decisions such as changing the status or levels of care based on evidence. Our study was conducted to support the LHS administration to plan for the human resources for health requirements for the upgraded hospital from a health centre.

Other results exhibit similar characteristics that have been documented in other countries where CHWs are used to undertake roles that are not their core cadres and tasks are informally shifted and shared without policies to ensure its rationality. This is mainly due to staff shortages $[19,20]$. The need for formalizing task shifting/sharing in situations of scarcity such as where medical officers, nursing officers and midwives are scarce is critical. The need for a task shifting and sharing policy with guidelines to ensure quality of services is important. Capacity building of these staff should be provided and 
continuous supervision to ensure tasks for the new roles are effectively provided is emphasized [20,21].

The results provide evidence that calls upon the health managers in PNG to review the role of community health workers in the health facilities. The work they undertake in the facility is far beyond their training and they tend to be easily translated into any cadre where need arises. Moreover, Braun District hospital is located in an island with no health facilities nearby thus the need for releasing the community health workers to undertake their core roles at the household levels with promotive, preventive and rehabilitative services as necessary.

Multitasking was reported among the non-clinical staff. Multitasking can have detrimental effects on task performance and increase errors. Conducting more than one task together may affect performance and increase cost in terms of decreased accuracy [22, 23] and increased reaction time to environmental stimuli [24]. Experimental studies have typically investigated this by presenting two tasks in close proximity and observing the participant's response to both. Some of the tasks do not require full-time staff but rather outsourcing when such services are needed like the electrician and the painter. These are not activities to be conducted on daily basis. Likewise, for clinical service, multitasking has effects on the quality and time taken to conduct the task. Increasing the time between the first and the second task reduces the delay in responding to the second task. Voluntarily or internally prompted, multitasking has different implications for efficiency and errors than when externally prompted [25].

There is no doubt that the WISN tool provides a scientific method of calculating staff workloads. However, there were limitations encountered during the study. These included under recording of service statistics, the eNHIS does not capture all the tasks provided by the experts but only reports on the key indicators identified for various programmes. This was mitigated by visiting the health facility to collect raw data from the daily registers. Some of the statistics conducted by similar cadres were aggregated and it was a challenge to apportion service statistics to specific cadres and thus a percentage was used.

\section{Conclusion}

The results from this WISN study provide evidence for basing various staffing decisions on. Some of the decisions include staff reassignment; some with surplus could mean anticipation of more clients considering the facility change of status as well staff prioritization for hiring. WISN method is an important methodology to help managers make important decisions such as changing the status of a facility like in this study; from a health centre to a district hospital.

\begin{abstract}
Abbreviations
AWT: Available working time; CHWs: Community health workers; HEO: Health extension officer; PNG: Papua New Guinea; eNHIS: Electronic National Health Information systems; WISN: Workload indicators of staffing need; LHS: Lutheran health services.
\end{abstract}

\section{Acknowledgements}

We acknowledge the support of many individuals who ensured that the WISN implementation went on smoothly. Special thanks to the NDoH Senior Executive Management team for endorsing this activity and the National Secretary for Lutheran Health Services Mr. Katu Yapi for the support and logistics provided. We appreciate WHO for the technical support provided and the Health Workforce Standards and Accreditation Branch team for the stewardship of this process. The health management team and staff of Braun District Hospital cannot be forgotten for their support.

\section{About this supplement}

This article has been published as part of Human Resources for Health Volume 19, Supplement 1 2021: Countries' experiences on implementing WISN methodology for health workforce planning and estimation. The full contents of the supplement are available at https://human-resourceshealth.biomedcentral. com/articles/supplements/volume-19-supplement-1.

\section{Authors' contributions}

DD and $M O$ conceived the idea. NM, MTA, TA, GTP, VJL, RJ, and PK conducted the field visit and collected the data. MO, DD, NM, MTA analysed the data and the produced the draft report. DL, AM, KY, RM and D reviewed the draft and produced a final document. All authors read and approved the final manuscript.

\section{Funding}

All the funding for this study from the design, field visit, data collection, analysis and interpretation of data was fully funded by LHS and WHO.

\section{Availability of data and materials}

Data and materials are available on request from NDoH and LHS report.

\section{Declarations}

Ethics approval and consent to participate

The NDoH endorsed the WISN methodology for all staffing requirements estimation and projections in 2017 thus all ethical approvals were waived by the $\mathrm{NDOH}$. The LHS in conformance requested the implementation of the WISN methodology to support the estimation of staffing levels in the newly upgraded hospital. Explanation on the need for the study was explained to the staff to ensure their participation.

Consent to publication

Not applicable.

Competing interests

All authors declare no competing interests.

\section{Author details}

${ }^{1}$ Health Workforce Standards and Accreditation Branch, National Department of Health, Port Moresby, Papua New Guinea. ${ }^{2}$ World Health Organization, Port Moresby, Papua New Guinea. ${ }^{3}$ Lutheran Health Services, Port Moresby, Morobe Province, Papua New Guinea.

Received: 13 October 2021 Accepted: 14 October 2021

Published: 28 January 2022 


\section{References}

1. Papua New Guinea-National Health Administrative Act, 1997.

2. PNG. Organic Law on Provincial Governments and Local level governments, NCD. 1997

3. Provincial Health Authority Act, 2007.

4. GoPNG. National Health Plan 2011-2020. 2010

5. Standard Treatment for Common Illnesses of Children in Papua New Guinea: A Manual for Nurses, Community Health Workers, Health Extension Officers and Doctors (8th Edition).

6. NDoH. National Human resources for Health Policy. 2012

7. NDoH. Free Primary Health care and subsidized specialised services Policy. 2013.

8. Global strategy on human resources for health: workforce 2030. Geneva: World Health Organization; 2016. http://www.who.int/hrh/resources/ 16059_Global_strategyWorkforce2030.pdf. Accessed 23 Nov 2020.

9. Human resources for health country profiles: Papua New Guinea. http:// iris.wpro.who.int/handle/10665.1/14596. Accessed 07 Oct 2020.

10. Workload Indicators of Staffing Need (WISN). User's manual. Geneva. 2010. Accessed 23 Nov 2020.

11. De Sousa YG et al. Work loads of nursing professionals in hospital services for mental health: an integrative review. J Health, Popul Nutr 2016;pp.1-9.

12. Shivam S, et al. Nursing personnel planning for rural hospitals in Burdwan District, West Bengal, India, using workload indicators of staffing needs. J Health Popul Nutr. 2014;32(4):658-64.

13. National Health Service Standards for Papua New Guinea-2010-2020 (Vol. 1 \&Vol 2).

14. Dawson A, Howes T, Gray N, Kennedy E. Human resources for health in maternal, neonatal and reproductive health at community level: a profile of Papua New Guinea, Human Resources for Health Knowledge Hub and Burnet Institute, Sydney, 2011.

15. Organization for economic cooperation and development: towards highperforming health systems. 2004, Paris: OECD.
16. Zurn P, Dal Poz MR, Stilwell B, Adams O. Imbalance in the health workforce. Hum Resour Health. 2004;2:13. https://doi.org/10.1186/ 1478-4491-2-13.

17. NDoH PNG 2016, Health Sector Review 2016. National Department of Health, Government of Papua New Guinea.

18. Liljamo P, Lavander P, Kejonen P. Determining optimal nursing resources in university hospital nursing officer staffing. Nurs Inform. 2016;2016:3.

19. Ly A, Kouanda S, Ridde V. Nursing and midwife staffing needs in maternity wards in Burkina Faso referral hospitals. Hum Resour Health. 2014;12:S8. https://doi.org/10.1186/1478-4491-12-S1-S8.

20. Dharmayuda A. Workload analysis of doctors at Puskesmas using workload indicators of staffing need in Denpasar. Publ Health Prev Med Arch. 2015:3:20-6.

21. Satish N. Gap analysis in staffing using workload indicators of staffing need method in a tertiary care teaching hospital. Int J Sci Res. 2015

22. Ouedraogo $L$, et al. Effectiveness of task sharing and task shifting on the uptake of family planning in Kenya. Adv Reprod Sci. 2020. https://doi.org/ 10.4236/arsci.2020.84018.

23. Adler and Benbunan-Fich, 2012. The effects of positive and negative selfinterruptions in discretionary multi-tasking.

24. Bakiri S, et al. Distraction and driving: results from a case-control responsibility study of traffic crash injured drivers interviewed at the emergency room. Accid Anal Prev. 2013;59:588-92.

25. West R, Scolaro AJ, Bailey K. when goals collide: the interaction between prospective memory and task switching. Can J Exp Psychol. 2011;65(1):38.

\section{Publisher's Note}

Springer Nature remains neutral with regard to jurisdictional claims in published maps and institutional affiliations.
Ready to submit your research? Choose BMC and benefit from:

- fast, convenient online submission

- thorough peer review by experienced researchers in your field

- rapid publication on acceptance

- support for research data, including large and complex data types

- gold Open Access which fosters wider collaboration and increased citations

- maximum visibility for your research: over $100 \mathrm{M}$ website views per year

At $\mathrm{BMC}$, research is always in progress.

Learn more biomedcentral.com/submissions 